\section{Detecção precoce de ototoxicidade usando emissões otoacústicas produtivas de distorção}

\author{
Juan C. Vallejo ${ }^{1}$, Mercedes N. Silva ${ }^{I}$, \\ Jose A. A. de Oliveira ${ }^{2}$, João J. Carneiro ${ }^{3}$, \\ Luiz S. O. Rocha ${ }^{4}$,Jose F. C. Figueiredo',
} Maria F. V.Chiosi ${ }^{6}$

Resumo

I

ntrodução: Os aminoglicosídeos são potencialmente ototóxicos, sendo que a monitorização tradicional detecta ototoxicidade quando a lesão já aconteceu. Objetivo: Estudamos prospectivamente a audição de pacientes que recebiam amicacina, usando audiometria tonal liminar (ATL) e emissões otoacústicas, produtos de distorção (EOA-PD), visando a suspeita precoce de ototoxicidade e sua prevenção. Forma de estudo: Prospectivo randomizado. Material e método: Avaliamos, com ATL, 29 pacientes em tratamento com amicacina, para detectar alterações audiométricas. Desses 29 pacientes, três tiveram alteração na ATL em 6 e/ou 8 kHz (10,34 \%) - um com alteração bilateral; os outros dois, unilateral (quatro ouvidos), todos assintomáticos. Realizamos EOA-PD, para estudar a função coclear. Dos 29 pacientes ( 58 ouvidos), nove (18 ouvidos) foram afastados do estudo por não completarem as testagens das EOA-PD, ficando 20 pacientes ( 40 ouvidos) para análise. Resultados: Não encontramos alterações significativas nas respostas das EOA-PD durante o vale e o pico sérico da amicacina, como esperado. Quando comparados os ouvidos com e sem alterações audiométricas, as variações das respostas não foram significativas em 1,2 e 4 kHz; no entanto, em 6 e $8 \mathrm{kHz}$ mostraram diferenças significativas $(p<0,05)$. As respostas das EOA-PD nos ouvidos com ATL normal apresentaram incrementos mais do que quedas; e o contrário aconteceu nos ouvidos que apresentaram ATL alterada, ou seja: houve mais quedas do que incrementos. Conclusões: a ototoxicidade pode ser monitorada eficazmente com EOA-PD durante a administração da droga, independentemente do horário da sua administração; os incrementos nas respostas poderiam ser um sinal precoce de ototoxicidade por aminoglicosídeos.

\section{Ototoxicity early detection using distortion product otoacoustic emissions}

Palavras-chave: emissões otoacústicas, ototoxicidade, detecção precoce.

Key words: otoacustic emissions, ototoxicity, monitoring.

\section{Summary}

\section{I}

ntroduction: Aminoglycosides can lead to ototoxicity, but the traditional auditory monitoring detects ototoxicity just when the injury has already occurred. Aim: A prospectively study of the auditory function of patients in treatment with amicacyn using pure tone audiometry (TA) and distortion product otoacoustic emissions (DPOAE) to detect an early suspicion of ototoxicity and its prevention. Study design: Prospective randomized. Material and method: Twenty-nine patients treated with amicacyn were evaluated with TA to detect audiometric changes. Out of 29 patients, three had audiometric changes in 6 and/or 8 $\mathrm{kHz}(10.34 \%)$, one had bilateral and two had unilateral alteration (four ears). They were all asymptomatic. We performed DPOAE to study the cochlear function. Out of 29 patients ( 58 ears), nine (18 ears) were excluded because they did not have all DPOAE tests, remaining 20 patients (40 ears) to analyze. Results: No specific tendency was found in the DPOAE records during the serum trough and peak of the drug. In a comparative study of ears with and without audiometric changes, no significant difference was found in the response in 1, 2 and $4 \mathrm{kHz}$; however, a significant difference was observed in 6 and $8 \mathrm{kHz}(p<0.05)$. We found that DPOAE responses of normal ears presented a larger number of increments than decreases when compared to the others. Conclusions: Ototoxicity can be effectively monitored with DPOAE during drug administration, regardless of time of administration, and 6 and $8 \mathrm{kHz}$ are more sensitive frequencies to realize effective auditory monitoring; the increments in responses can be an early sign of ototoxicity by aminoglycosides.

\footnotetext{
${ }^{1}$ Médico Adido do Departamento de Otorrinolaringologia do HCFMRP-USP.

${ }^{2}$ Professor Titular e Chefe do Departamento de Otorrinolaringologia do HCFMRP-USP

${ }^{3}$ Docente do Departamento de Cirurgia do HCFMRP-USP.

${ }^{4}$ Médico Contratado da Comissão de Uso e Controle de Antimicrobianos do HCFMRP-USP

${ }^{5}$ Docente do Departamento de Clínica Médica, Disciplina de Molestias Infecciosas do HCFMRP-USP.

${ }^{6}$ Médico Residente do Departamento de Clínica Médica do HCFMRP-USP.

Departamento de Oftalmologia e Otorrinolaringologia do Hospital das Clínicas da Faculdade de Medicina de Ribeirão Preto - Universidade de São Paulo.
}

Endereço para correspondência: Prof. Dr. José Antônio Apparecido de Oliveira - Departamento de Oftamologia e Otorrinolaringologia do Hospital das Clínicas de Ribeirão Preto - Av. Bandeirantes 3900 - 14096-900 Ribeirão Preto /SP. Telefone.: (0xx16) 602-2523 - Fax: (0xx16) 602-2860.

Artigo recebido em 03 de abril de 2001. Artigo aceito em 17 de abril de 2001. 


\section{INTRODUÇÃO}

A utilização de fármacos ototóxicos na prática clínica é ampla e nem sempre a possibilidade de uma alteração na função auditiva é seriamente considerada. Os aminoglicosídeos podem causar lesões progressivas no epitélio sensorial auditivo a partir das espiras basais, até as apicais, sendo as células ciliadas externas (CCE) as atingidas ${ }^{8}$. A amicacina é um antibiótico aminoglicosídeo muito usado com propriedades ototóxicas conhecidas, sendo que a sua potencial toxicidade é predominantemente coclear ${ }^{8}$.

A incidência geral de ototoxicidade causada por antibióticos aminoglicosídeos é muito variável e vai de $5 \%$ até 33\%, dependendo da droga usada, dose, tempo de administração, condição do paciente, suscetibilidade individual e até a metodologia de avaliação $\mathrm{O}^{2,7,8,10,20}$.

Existem várias técnicas para monitorar a função auditiva durante a administração de ototóxicos $3,4,22,26,30$. A audiometria tonal liminar (ATL) tem sido tradicionalmente usada com esse propósito ${ }^{22}$. A audiometria de altas freqüências também tem sido usada; porém, não está ao alcance de todos e não existe consenso em relação aos critérios de interpretação ${ }^{3}$. Os dois tipos de audiometrias apresentam resultados subjetivos, tanto em relação ao paciente quanto ao examinador. A condição do paciente torna-se fator limitante, pela necessidade de ambiente sem ruído. Na prática clínica, as monitorizações da função auditiva durante tratamentos com ototóxicos são feitas usando a ATL na procura de aumentos progressivos dos limiares ${ }^{26}$.

As emissões otoacústicas (EOA), que registram os mecanismos cocleares ativos atribuídos principalmente à função das $\mathrm{CCE}^{15}$, podem ser usadas durante o uso de ototóxicos, para estudar a função coclear ${ }^{9,13,18,19,26}$. O registro das EOA, usado com esse propósito, tem demonstrado alteração das respostas antes que sejam registradas alterações no limiar auditivo ${ }^{14}$. As EOA têm maior especificidade e sensibilidade do que os outros métodos na avaliação da função auditiva, sendo isso uma importante vantagem em relação às outras técnicas mencionadas ${ }^{26}$.

O objetivo deste trabalho foi estudar a função auditiva de pacientes em tratamento com amicacina, através de ATL e EOA-PD, para determinar a ocorrência ou não de ototoxicidade e para avaliar potenciais alterações na fisiologia do ouvido interno - e detectar precocemente toxicidade coclear.

\section{MATERIAL E MÉTODO}

\section{Sujeitos}

A função auditiva de 32 pacientes de ambos os sexos, com idade entre 18 e 50 anos, foi avaliada prospectivamente durante internação hospitalar, ocasião em que recebiam tratamento antibiótico com amicacina por via intramuscular a $500 \mathrm{mg}$ de $12 \mathrm{em} 12$ horas, durante sete dias, com fins terapêuticos.
Antes de iniciar o estudo, os pacientes foram informados sobre sua participação e assinaram um termo de consentimento.

\section{Testes audiométricos}

ATL e impedanciometria foram realizados nos pacientes, previamente ao tratamento e 15 dias após seu término. A ATL foi feita nas freqüências de 0,25, 0,5, 1, 2, 4, 6 e $8 \mathrm{kHz}$. Esses testes foram realizados no setor de Fonoaudiologia do Hospital usando um equipamento Maico MA-41, para a ATL, e um Interacoustics AZ-7, para a impedanciometria.

Com essa avaliação audiométrica, determinamos nos pacientes a presença de uma audição normal prévia à inclusão no estudo e a ocorrência ou não de alterações auditivas após a administração da amicacina. O critério usado para determinar alteração auditiva por ototoxicidade foi um incremento igual ou maior que $15 \mathrm{~dB}$ NA no limiar audiométrico, em uma ou mais freqüências. Este é um critério para ototoxicidade aceito por vários autores na literatura revisada $3,5,17,21$.

Previamente às testagem das EOA-PD, os pacientes foram interrogados em relação à presença de hipoacusia, acúfenos e vertigem como manifestações de ototoxicidade, e foi realizada otoscopia na investigação de alterações sugestivas de patologia de ouvido médio. Todos os testes com EOA durante o tratamento foram feitos com o paciente no leito.

\section{Emissões otoacústicas}

As testagens das EOA-PD foram realizadas nas freqüências de 1, 2, 4, 6 e $8 \mathrm{kHz}$, antes, durante e após o tratamento. Durante o tratamento, os testes foram feitos nos últimos quatro dias da administração da droga, no vale (antes da dose seguinte) e no pico sérico da amicacina (uma hora após administração) 5,12,17. Finalmente, os pacientes foram testados 15 dias depois de terminado o tratamento.

No modelo de estudo, prescindimos das dosagens séricas da amicacina no sangue, porque nosso objetivo foi verificar se existia diferença nas respostas das EOA-PD entre esses dois momentos séricos, independentemente de os níveis séricos da droga durante o pico e o vale serem ou não normais.

$\mathrm{Na}$ realização dos testes das EOA, utilizamos um equipamento ILO 92 Otodynamics, com sonda A (adulto).

\section{Testes laboratoriais}

Uma dosagem sérica de creatinina foi realizada em todos os pacientes, para avaliar a função renal.

\section{Critérios de inclusão}

- Pacientes de ambos os sexos com idade entre 18 e 50 anos. 
- Pacientes sem antecedentes de patologia otológica ou outra condição que pudesse alterar a função auditiva.

- Pacientes com audiometria, impedanciometria e EOA normais.

- Pacientes submetidos a todas as testagens propostas com EOA - antes, durante e após o tratamento.

- Pacientes que, durante o tratamento, não apresentassem sinais otoscópicos de patologia de ouvido médio.

- Pacientes submetidos à antibioticoterapia pelo tempo descrito.

- Termo de consentimento explicado, aceito e assinado pelo paciente.

\section{Análise dos resultados}

A análise dos resultados foi feita conforme a seguir:

1. Cálculo da incidência de alterações na ATL nos pacientes, segundo o critério usado.

2. Estudo por freqüência das respostas EOA-PD, durante os picos e vales séricos da droga.

3. Estudo comparativo por freqüência das variações das EOA-PD, entre os ouvidos com e sem alterações audiométricas, tomando como referência o teste inicial.

4. Estudo, durante o tratamento, do comportamento das EOA-PD, analisando o total dos casos e também os ouvidos com e sem alterações na ATL, separadamente.

A análise dos resultados com as EOA-PD foi feito por ouvidos, pela necessidade de saber o que aconteceu em cada cóclea de forma independente. Essa forma de análise já está padronizada nesse tipo de estudos.

A presença de sintomas como hipoacusia, vertigem e zumbidos, assim como a função renal dos pacientes, foi considerada em todas as análises.

A realização deste estudo foi aprovada pelo Conselho de Ética em Pesquisa do Hospital das Clínicas da Faculdade de Medicina de Ribeirão Preto, da Universidade de São Paulo. Todos os procedimentos foram cuidadosamente realizados seguindo as diretrizes do Conselho Nacional de Saúde, referentes às normas de pesquisa envolvendo seres humanos que constam na Resolução CNS 196/96.

\section{RESULTADOS}

De 32 pacientes testados, 29 completaram os testes audiométricos. Desses 29 pacientes, após ter sido realizada a ATL, três apresentaram alterações na ATL (segundo o critério adotado) e os restantes 26 não registraram mudanças significativas no limiar audiométrico. Essas alterações foram observadas nas freqüências de 6 e/ou 8 kHz. Dos três pacientes com alteração audiométrica, um deles teve alteração bilateral; e os outros dois, unilateral, ou seja: foram quatro os ouvidos alterados (Tabela 1). Esse resultado define uma incidência de problema auditivo de 10,34\% dos pacientes (Figura 1).
Todos os casos que apresentaram alteração auditiva foram assintomáticos quanto a hipoacusia, zumbido ou vertigem.

Tabela 1. Descrição dos casos com alteração audiométrica

\begin{tabular}{lcc}
\hline Paciente & Lado afetado & Freqüência \\
\hline № 3 & Bilateral & 6 e $8 \mathrm{kHz}$ \\
№ 7 & Esquerdo & $8 \mathrm{kHz}$ \\
№ 13 & Esquerdo & $8 \mathrm{kHz}$ \\
\hline
\end{tabular}

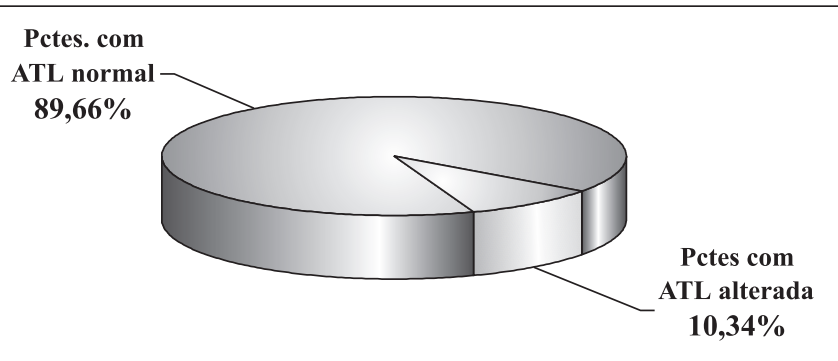

Figura 1. Resultado da Audiometria

Dos 29 pacientes (58 ouvidos), nove (18 ouvidos) foram afastados do estudo das EOA por não terem todas as testagens propostas, ficando um total de 20 pacientes (40 ouvidos) a estudar.

Já na análise das EOA-PD, não foram encontradas variações significativas nas respostas durante o vale e o pico sérico da droga. De modo geral, as respostas durante os picos não foram menores do que durante os vales séricos da amicacina, como era esperado - mas, em relação à resposta do teste inicial, tenderam a cair (Tabelas 2 e 3).

Quando comparados os ouvidos com e sem alterações audiométricas, não houve diferença significativa das médias das respostas das EOA-PD em 1, 2 e 4 kHz; no

Tabela 2. Médias das variações das respostas das EOA-PD nos picos e vales séricos da amicacina (em dB NPS) nos pacientes com ATL normal

\begin{tabular}{lcccccc}
\hline & Inicial & $1^{\mathrm{a}}$ (vale) & $2^{\mathrm{a}}$ (pico) & $3^{\mathrm{a}}$ (vale) & $4^{\mathrm{a}}$ (pico) & Final \\
\hline $1 \mathrm{kHz}$ & 0 & 0,53 & 0,04 & 0,32 & 0,7 & $-0,13$ \\
$2 \mathrm{kHz}$ & 0 & 0,20 & $-0,32$ & 0,12 & $-0,35$ & $-0,18$ \\
$4 \mathrm{kHz}$ & 0 & $-0,92$ & $-0,72$ & $-0,40$ & $-0,85$ & $-0,29$ \\
$6 \mathrm{kHz}$. & 0 & $-0,002$ & 0,16 & $-0,16$ & $-0,57$ & 0,23 \\
$8 \mathrm{kHz}$. & 0 & 1,04 & 0,88 & 1,4 & 0,5 & 1,10 \\
\hline
\end{tabular}

Tabela 3. Médias das variações das respostas das EOA-PD nos picos e vales séricos da amicacina (em dB NPS) nos pacientes com ATL alterada

\begin{tabular}{lcccccc}
\hline & Inicial & $1^{\mathrm{a}}$ (vale) & $2^{\mathrm{a}}$ (pico) & $3^{\mathrm{a}}$ (vale) & $4^{\mathrm{a}}$ (pico) & Final \\
\hline $1 \mathrm{kHz}$ & 0 & $-0,77$ & $-1,62$ & $-0,2$ & $-1,1$ & $-2,8$ \\
$2 \mathrm{kHz}$ & 0 & $-1,05$ & $-1,6$ & $-1,875$ & $-2,075$ & $-3,15$ \\
$4 \mathrm{kHz}$ & 0 & 0,3 & $-3,67$ & $-4,57$ & $-1,47$ & $-3,37$ \\
$6 \mathrm{kHz}$. & 0 & $-4,4$ & $-2,05$ & $-2,6$ & $-2,62$ & $-3,65$ \\
$8 \mathrm{kHz}$. & 0 & $-5,9$ & $-3,12$ & $-10,52$ & $-3,17$ & $-4,47$ \\
\hline
\end{tabular}


entanto, em 6 e $8 \mathrm{kHz}$ essas diferenças foram significativas $(p<0,05)$. Esses dados estão detalhados na Tabela 4 e representados na Figura 2.

No decorrer do estudo, além das quedas, observamos incrementos nas respostas das EOA-PD. Esses incrementos variaram em intensidade, desde pequenos e pouco significantes a grandes incrementos, que chegaram até 9 dB NPS acima do nível do teste inicial. Esse fenômeno, no total de pacientes, aconteceu em 55\% dos ouvidos.
Tabela 4. Médias das variações das EOA-PD (en dB NPS)

\begin{tabular}{lccccc}
\hline & \multicolumn{2}{c}{ Pacientes com ATL normal } & & \multicolumn{2}{c}{ Pacientes com ATL alterada } \\
\cline { 2 - 3 } \cline { 5 - 6 } & Média & D.P. & & Média & D.P. \\
\hline $1 \mathrm{kHz}$ & -0.11 & 6.33 & & -1.3 & 2.7 \\
$2 \mathrm{kHz}$ & -0.11 & 2.97 & & -1.95 & 2.8 \\
$4 \mathrm{kHz}$ & $-0,64$ & 3,31 & & -2.56 & 3.3 \\
$6 \mathrm{kHz}$. & 0,06 & 3,58 & & -3.05 & 2.78 \\
$8 \mathrm{kHz}$. & 0.99 & 5.68 & & -5.44 & 6,26 \\
\hline
\end{tabular}

$p<0,05$
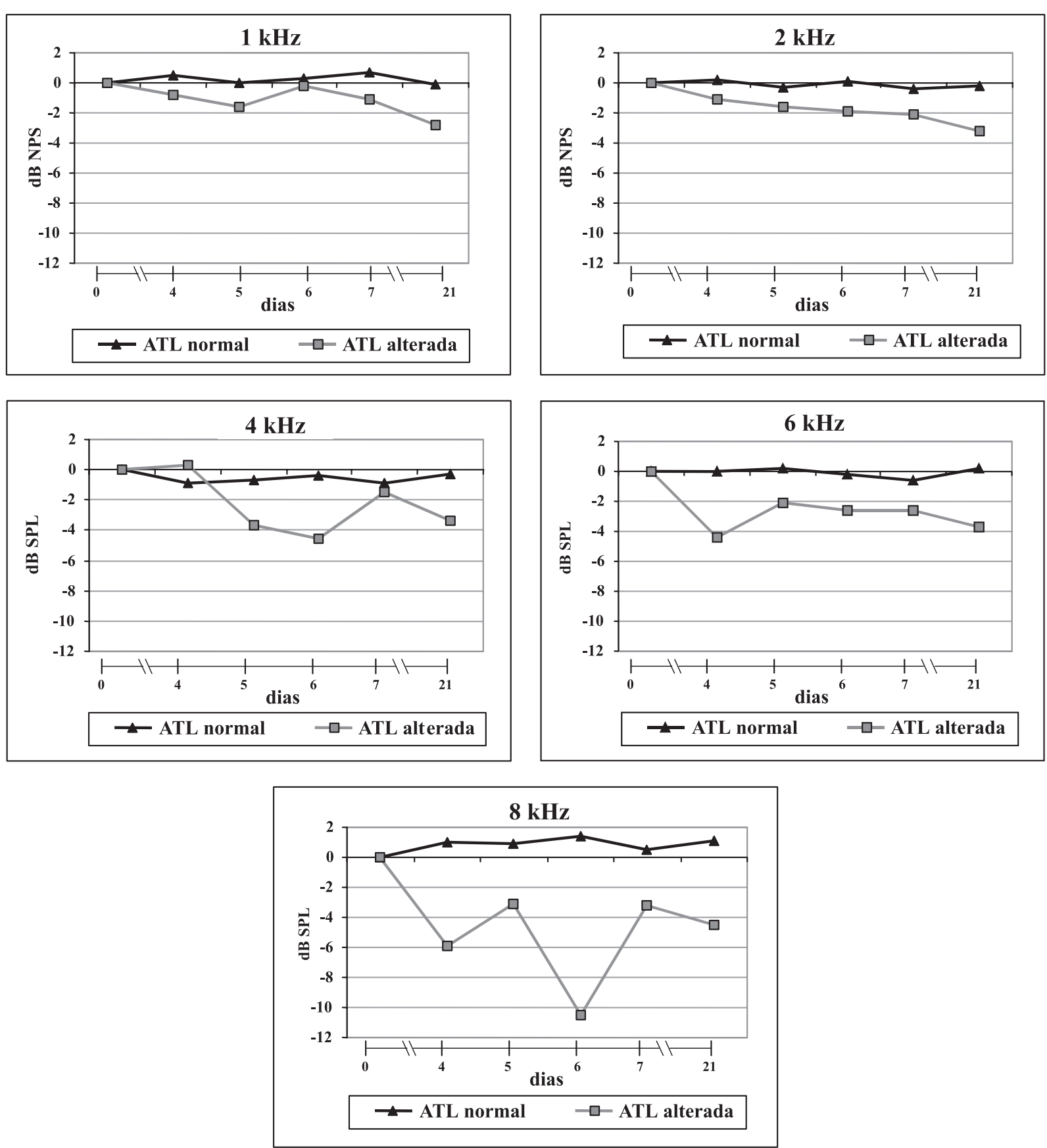

Figura 2. Variações das respostas das EOA-PD por freqüência 
Nos ouvidos sem alteração audiométrica, de um total de 180 testagens, em 106 (59\%) houve incremento na resposta; e nos 74 restantes (41\%) houve queda. Nos ouvidos que tiveram alterações na ATL, de 20 testagens, em três (15\%) houve incremento e nas outras 17 (85\%) houve queda (Tabela 5). A diferença entre estes dois casos foi significativa $\left(\chi^{2}=13,98 ; p<0,0005\right)$ (Figura 3).

Os valores de creatinina sérica permaneceram dentro da normalidade em todos os pacientes durante a administração da amicacina

Tabela 5. Distribuição dos casos que apresentaram incrementos ou quedas nas respostas das EOA-PD

\begin{tabular}{lcc}
\hline & $\begin{array}{c}\text { Pacientes com ATL } \\
\text { normal }\end{array}$ & $\begin{array}{c}\text { Pacientes com ATL } \\
\text { alterada }\end{array}$ \\
\hline Incrementos & $106(59 \%)$ & $3(15 \%)$ \\
Quedas & $74(41 \%)$ & $17(85 \%)$ \\
\hline
\end{tabular}

$\chi^{2}=13,98$

$p<0,0005$

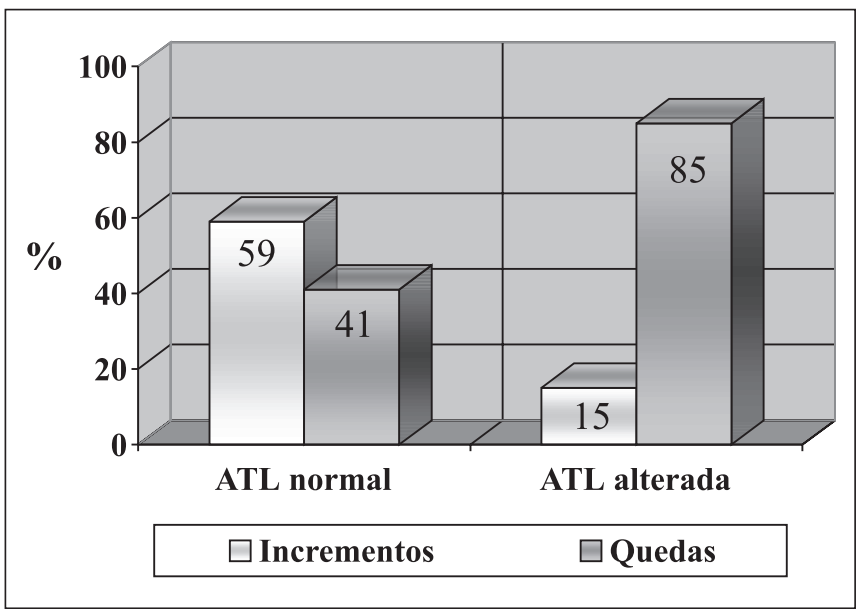

Figura 3. Incrementos e quedas das respostas das EOA-PD

\section{DISCUSSÃO}

As EOA permitem medir a atividade mecânica das $\mathrm{CCE}^{15}$ - um teste totalmente objetivo, pois as respostas são independentes da vontade do paciente, e isso permite a avaliação em qualquer idade. As drogas ototóxicas podem potencialmente alterar a biomecânica coclear afetando a audição ${ }^{24}$. Sendo asim, o registro das EOA é útil na avaliação da função auditiva em pacientes que estão recebendo drogas ototóxicas, sendo mais sensível do que outros métodos ${ }^{26}$. Kakigi e outros autores sugerem que as EOA-PD são mais precisas do que as EOA transientes para monitorar em locais específicos a função das CCE, e que essas respostas alteradas podem representar pequenas regiões de CCE lesadas ${ }^{12,28}$.
A incidência geral de ototoxicidade é variável, dependendo de alguns fatores. Brummett cita uma incidência de 20-33\%; e, segundo ele, ela varia dependendo dos critérios usados para ototoxicidade e das circunstâncias que envolvem as avaliações audiométricas. Conclui o autor que essa incidência pode ser exagerada ${ }^{2}$. Para Gutierrez, têm que ser considerados os fatores de risco para ototoxicidade, como o potencial ototóxico intrínseco do fármaco, alterações da função renal, administração simultânea de outros medicamentos ototóxicos, dose acumulativa total da droga, idade avançada e exposição a ruído. Esse autor encontrou uma incidência de ototoxicidade de 13,9\% para amicacina, de 3,3\% para gentamicina, de $6,1 \%$ para tobramicina e de 2,4\% para netilmicina ${ }^{8,25}$. Matz, em estudo prospectivo, avaliou os efeitos ototóxicos em pacientes recebendo amicacina, gentamicina, netilmicina e tobramicina, encontrando uma incidência de 11\%, 12,9\%, 11,5 e $2 \%$, respectivamente ${ }^{20}$. Esses e outros estudos demonstram a grande variabilidade da ototoxicidade desses fármacos ${ }^{5,29}$.

No nosso estudo, encontramos que a incidência de ototoxicidade foi de 10,34\%, envolvendo as freqüências de $6 \mathrm{e} / \mathrm{ou} 8 \mathrm{kHz}$, similarmente ao descrito na literatura.

Gordon e colaboradores afirmam que a ototoxicidade clinicamente aparente é incomum: provavelmente ocorre em 1\% dos casos, quando a terapia é mantida no tempo mínimo. Com monitoramentos mais sensíveis, perdas auditivas em altas freqüências são mais comuns de serem notadas ${ }^{6}$. No nosso trabalho, os pacientes permaneceram assintomáticos, provavelmente porque as freqüências afetadas foram de 6 e $8 \mathrm{kHz}$. Por esse motivo, é importante ter presente que o uso desse tipo de antibióticos, mesmo por curto tempo, pode induzir ototoxicidade subclínica.

Nas testagens, com EOA-PD, realizadas durante os picos e os vales séricos da amicacina no sangue, não observamos variações significativas nas respostas das EOA-PD nesses dois momentos - e isso sugere que os níveis máximos e mínimos da droga no sangue não estão associados a mudanças das respostas das EOA-PD. Pelos nossos achados, acreditamos que durante o tratamento com amicacina o horário da realização dos testes parece não ser fundamental.

Alguns autores descrevem que pacientes que se encontram sob regime de aminoglicosídeos apresentam as respostas das EOA-PD prejudicadas: essas respostas vão caindo na razão direta do tempo de tratamento ${ }^{3,9,16}$. No nosso trabalho, comparando a resposta inicial com as respostas subseqüentes, observamos diferenças dependendo do caso. Assim, nos ouvidos sem alteração audiométrica as respostas não caíram significativamente em qualquer uma das freqüências. Isso não aconteceu nos ouvidos com ATL alterada: nesses casos, as quedas nas respostas aconteceram em todas as freqüências - e essas quedas 
foram maiores nas freqüências mais altas. Quando comparadas as médias dessas quedas entre os dois casos, observamos que apenas nas freqüências de 6 e $8 \mathrm{kHz}$. a diferença foi significativa $(p<0,05)$ (Tabela 4). Isso significa que a função coclear nos ouvidos com alteração audiométrica ficou mais alterada do que nos outros, apesar de em todos os tratamentos ter sido idêntica. Essas alterações das EOA-PD aconteceram nas mesmas freqüências, onde os limiares auditivos medidos na última ATL mostraram queda (6 e $8 \mathrm{kHz}$ ), o que comprova que tanto a ATL quanto o registro das EOA-PD são métodos eficientes na detecção de alterações auditivas decorrentes da administração de ototóxicos.

Durante a realização das testagens, observamos como fenômeno constante incrementos das respostas das EOA-PD, ao invés de diminuições, como seria o esperado. Igual fenômeno temos observado em pesquisas (ainda não publicadas) em animais de experimentação. Esse fato já foi descrito em 1986, por Neely e Kim³; e por Brown e colaboradores $^{1}$, em 1988. Mais recentemente, Mulheran e Degg $^{22}$, Kakigi e colaboradores ${ }^{11}$ e Raveh $^{27}$ estudaram sistematicamente esse fenômeno, e dão grande importância às suas implicações patológicas, alertando que pode tratarse de um sinal inicial de lesão coclear por uso de aminoglicosídeos. Concordam com a hipótese que explica esse fenômeno e afirmam que se trata de uma disfunção ou lesão coclear na área vizinha à da resposta. Essa lesão setorial favorece as condições de captação do "som da resposta", através dessas regiões cocleares "silenciosas", hipofuncionantes ou lesadas, fazendo que sejam captadas com maior facilidade pela sonda do aparelho. Apesar de não haver evidências, acreditamos que esse fenômeno poderia também resultar de uma irritação das CCE antes de lesão, similarmente ao que acontece nas células do sistema vestibular. De qualquer forma, esses dois possíveis mecanismos ainda estão no campo da hipótese.

No nosso estudo, encontramos que os incrementos aconteceram com maior freqüência nos ouvidos sem alteração audiométrica (59\%); já nos ouvidos com alteração na ATL, esses incrementos foram muito menos freqüentes (15\%), predominando as quedas (85\%); a diferença entre os dois casos foi significativa $\left(\chi^{2}=13,98 ; p<0,0005\right)$. Isso sugere o seguinte:

1. Os incrementos das respostas precedem às quedas.

2. Os incrementos das respostas aconteceriam antes de a cóclea sofrer lesão definitiva, anunciando a alteração.

3. As quedas, se significativas, estariam já denunciando uma lesão coclear definitiva.

\section{CONCLUSÕES}

Concluímos que os tratamentos feitos com ototóxicos, como a amicacina, podem causar lesão auditiva até subclínica, mesmo quando usados por períodos curtos; portanto, a avaliação dos sintomas auditivos é ineficaz para detectar o início da lesão, ou seja: não teria valor preventivo. A avaliação com EOA-PD constitui-se em um meio sensível para a detecção precoce da lesão coclear através dos registros dos incrementos e quedas das respostas durante um tratamento com ototóxicos.

Com base em alguns estudos ${ }^{3,8,9,28}$ e nos nossos achados, acreditamos que durante os tratamentos com ototóxicos é importante e efetivo o monitoramento com EOA-PD na detecção precoce de ototoxicidade, com especial atenção aos incrementos e quedas nas respostas das EOA-PD.

\section{BIBLIOGRAFIA}

1. BROWN, A.M.; McDOWELL, B.; FORGE, A.- Acoustic distortion products can be used to monitor the effects of chronic gentamicin treatment. Hearing Research, 36:143-56, 1989.

2. BRUMMETT, R.E.; MORRISON, R.B. - The incidence of aminoglycoside antibiotic-induced hearing loss. Arch Otolaryngol Head Neck Surg, 116:406-10, 1990

3. CAMPBELL, K.C.M., PhD; DURRANT, J., MD.- Audiologic monitiring for ototoxicity, In: Otolaryngologic Clinics of North America (Ototoxicity), 26:903-10, 1993.

4. COLleT, L.; VEUIllET, E.; CHANAL, J.M.; DESREUX, V.; MERMET, B.; DISANT, F.; MORGON, A. - Étude de l'ototoxicité de l'amikacine et de la nétilmicine au moyen des oto-émissions acoustiques provoquées et de l'audiométrie hautes fréquences. Pathologie Biologie, 40:990-2, 1992.

5. GATELL, J.M. et al. - Univariate and multivariate analyses of risk factors predisposing to auditory toxicity in pacients receiving aminoglycosides. Antimicrobial Agents and Chemotherapy, 31:1383-7, 1987.

6. GORDON, L.A.; RONALD, E.P. - In: FAUCI et al.- Harrison's Principles of Internal Medicine. 14 $4^{\text {th }}$. ed., New York, McGraw-Hill, 856-69, 1998.

7. GOVAERTS, P.J.; CLAES, J.; VAN DE HEYNING, P.H.; JORENS, P.G.; MARQUET, J.; DE BROE, M.E. - Aminoglycoside-induced ototoxicity. Toxicol Lett, 52:227-51, 1990.

8. GUTIERREZ, O. et al.- Aminoglucósidos y efecto post antibiótico. Rev Soc Mex Ped, 59:1-8, 1992.

9. HOTZ, M.A.; HARRIS, P., PhD; PROBST, R., MD.- Otoacoustic emissions: an aproach for monitoring aminoglycoside-induced ototoxicity. Laryngoscope, 104:1130-4, 1994.

10. KAHLMETER, G.; DAHLAGER, J.I.- Aminoglycoside toxicity - a review of clinical studies published between 1975 and 1982. J. Antimicrob Chemother, 13 Suppl A:9-22, 1984.

11. KAKIGI, A. et al. - Basal cochlear lesions resutl in increased amplitude of otoacoustic emissions. Audiol Neurootol, 3:361-72, 1998.

12. KAKIGI, A; HIRAKAWA, H.; HAREL, N; MOUNT, R.J.; HARRISON, R.V. - Comparison of distortion product and transient evoked otoacoustic emissions with ABR threshold shift in chinchillas with ototoxic damage. Auris Nasus Larynx, 25:223-32, 1998.

13. KAYE, D., MD. - In: Davis M. The Merck Handbook. 9aㅡ edição, New York, Doyma, 25-50, 1990

14. KEMP, D.T. - Development in cochlear mechanics and techniques for noninvasive evaluation. Adv Audiol, 5:27-45, 1988.

15. KEMP, D.T. - Stimulates acoustic emissions from within the human auditory system. J Acoust Soc Am, 64:1386-91, 1978.

16. KUMAGAI, S. - Distortion-product otoacoustic emissions in kanamicintreated guinea pig cochlea. Nippon Jibiinkoka Gakkai Kaibo, 98:368-79, 1995.

17. LERNER, S., MD.; SMITH, B.; SELIGSON, R., MD. - Comparative study of ototoxicity and nefrotoxicity in pacients randomly assignes to treatment with amikacin or gentamicin. Am.J. Med., $80 \mathrm{suppl} 3 \mathrm{~B}$. 98-104, 1986. 
18. LONSBURY-MARTIN, B.L.; MARTIN, G.K. - The clinical utility of distortion-product otoacoustic emissions. Ear Hear, 11:144-54, 1990.

19. LOPES FILHO, O.; THOMÉ, D.; ECKLEY, C.- Emissões otoacústicas transitórias e produtos de distorção na avaliação da audição em recém nascidos com poucas horas de vida. Rev. Bras. de Otorrinolaringol., 62:220-8, 1996.

20. MATZ, G.J. - Aminoglycoside ototocicity. Am J Otolaryngol, 7:117-9, 1986.

21. MOORE, R.; SMITH, C.; LIETMAN, P. - Risk factors for the development of auditory toxicity in pacients receiving aminoglycosides. JInfectious Diseases. 149:23-30, 1984.

22. MULHERAN, M.; DEGG, C.- Comparison of distortion product OAE generation betwen a patient growth requirent frequent gentamicin therapy and control subjects. Br.J. Audiol., 31:5-9, 1997.

23. NEELY, S.T.; KIM, D.O. - A model for active elements in cochlear biomecanics. J Acoust Soc Am, 79:1472-80, 1986.

24. OLIVEIRA, J.A.A. - Em: COSTA, S.S.; CRUZ, O.L.E; OLIVEIRA, J.A.A. - Otorrinolaringologia: Princípios e Prática, 1a. ed., Porto Alegre RS, Artes Médicas, 95-97, 1994.
25. OLIVEIRA, J.A.A.- In: OLIVEIRA, J.A.A. - Audiovestibular Toxicity of Drugs. $1^{\text {st }}$ ed. Miami, C.R.C. Press, 87-91, 1989.

26. PROBST, R.; HARRIS, F.; HAUSER, R.- Clinical monitoring using otoacoustic emissions. Br.J. of Audiol, 27:85-90, 1993.

27. RAVEH., E.; MOUNT., R.J.; HARRISON, R.V. - Increased otoacousticemission amplitude secondary to cochlear lesions. J Otolaryngol, 27:354-60, 1998.

28. SHEHATA-DIELER, W.E.; DIELER, R.; TEICHERT, K.; MOSER, L.M. Intra and intersubject variability of acoustically evoked otoacoustic emissions II. Distortion product otoacoustic emissions. Laryngorbinootologie, 78:345-50, 1999.

29. WEINSTEIN, L. - In: GOODMAN L. \& GILMAN A. - The Pharmacologic Basis of Therapeutics. New York. Mcmillam. 1167-82, 1985.

30. ZOROWKA, P.; SCHMITT, H. - Evoked otoacoustic emissions and pure tone threshold audiometry in patients receiving therapy. Int.J. Ped. Otolar, 25:73-80, 1993 